\title{
ON THE VIBRATIONAL STABILITY OF GASEOUS STARS
}

\section{P. LEDOUX ${ }^{\mathrm{I}}$}

\section{ABSTRACT}

The dependence of the vibrational stability of stars upon their masses has been investigated, and it has been established that for laws of generation of energy of the type of Bethe's law, the ordinary law of opacity and a ratio of the specific heats of matter $\gamma$ equal to $\frac{5}{3}$, some instability would first appear for masses larger than $100 M_{\odot}$.

Changes in the law of opacity as well as in the ratio of the specific heats are more likely to occur in the outer layers of the stars, and their effects on the stability, especially for the giants, can be important.

If the region where they occur is appropriately chosen, these changes could render the star unstable for one of the higher modes (first or second), although it remains stable for the preceding ones. Some attention was also paid to the stability of "shell-source" and "ball-source" models with the conclusion that they would not exhibit any special instability.

I. The condition for the stability of stars for small radial pulsations was established for the case of radiative equilibrium by S. Rosseland, ${ }^{2}$ and T. G. Cowling ${ }^{3}$ obtained it for the case where a part of the star is in convective equilibrium. In Cowling's investigation the radiation pressure was treated as negligible (small mass) and the present author, ${ }^{4}$ in a paper which will be quoted as I, extended it to the case where the radiation pressure is appreciable.

If we define $A_{k}$ by

$$
\left.\begin{array}{r}
A_{k}=\int_{0}^{M} \frac{\delta \rho_{k}}{\rho}\left[\left(\Gamma_{3}-\mathrm{I}\right) \delta_{k}\left\{\epsilon_{\mathrm{I}}+\epsilon_{2}-\epsilon_{3}-\frac{d}{d m}\left[4 \pi r^{2}\left(F_{\mathrm{I}}+F_{3}\right)\right]\right\}\right. \\
\left.-\frac{2}{3} \delta_{k}\left[4 \pi r^{2} \bar{C}_{r} \frac{d P}{d m}+\epsilon_{2}+\frac{d}{d m}\left[4 \pi r^{2} F_{2}\right]\right]\right] d m
\end{array}\right\}
$$

the condition of stability of the star for its $k$ th mode of vibration is that $A_{k}$ be negative. In this expression, which is obtained by the ordinary method of perturbations, we have to introduce for the amplitudes $\delta r, \delta \rho, \delta T$, etc., their values corresponding to an adiabatic pulsation. The meaning of the different symbols is as follows: $\left(\Gamma_{3}-\mathrm{I}\right)$, defined 5 by $\delta T / T=\left(\Gamma_{3}-\mathrm{I}\right) \delta \rho / \rho$ for an adiabatic modification, is equal to $\left(\Gamma_{\mathrm{I}}-\beta\right) /(4-3 \beta)$, where $\beta$ is the ratio of the gas pressure $p_{G}$ to the total pressure $P=p_{G}+p_{R}$ and $\Gamma_{I}$, defined by $\delta P / P=\Gamma_{\mathrm{r}} \delta \rho / \rho$, has the well-known value

$$
\Gamma_{\mathrm{I}}=\beta+\frac{(4-3 \beta)^{2}(\gamma-\mathrm{I})}{\beta+\mathrm{I} 2(\gamma-\mathrm{I})(\mathrm{I}-\beta)}
$$

$\gamma$ is the ratio of the specific heats for the matter; $\epsilon_{\mathrm{I}}$ is the rate of generation of energy of subatomic origin per unit mass per second; $F_{\mathrm{I}}$ is the flux of radiation; $\bar{C}_{r}$ is the mean radial component of the velocity of turbulence at the point considered and has the value

I Fellow of the Belgian American Educational Foundation at the Yerkes Observatory.

${ }^{2}$ Oslo Pub., No. I, I931.

3 M.N., 96, 42, I936.

${ }_{4}^{4}$ Astrophysica Norvegica, 3, I93, I940.

5 S. Chandrasekhar, Introduction to the Study of Stellar Structure, p. 56, I939. 
given by equation (2) in $I ; \epsilon_{2}$ is the rate of transformation of the turbulent kinetic energy into thermal energy per unit mass per second, due to the viscosity, and its order of magnitude is given by $\epsilon_{2}=$ constant $\times q^{3} / l$, if $q$ is a mean velocity of turbulence and $l$ the mean free path of the elements in turbulent motion; $\epsilon_{3}=P \operatorname{div} \bar{C}_{r} / \rho$ is the rate of conversion of thermal energy into turbulent kinetic energy per unit mass per second; $F_{2}=\overline{\frac{1}{2} \rho C^{2} C_{r}}$ is the mean radial component of the vector representing convection of turbulent energy; and $F_{3}=\overline{\rho E C}_{r}$ is the mean radial component of the vector representing convection of thermal energy and has the value given by equation ( 3 ) in I.

However, this condition of stability is valid only if the deviations from an adiabatic pulsation remain small in the whole star. But it is well known ${ }^{6}$ that in a relatively small region near the surface $\left(T<6 T_{e}\right)$ this is not the case. However, as S. Rosseland ${ }^{7}$ has shown, in that region the amplitudes of the oscillations will adjust themselves in such a way that there will be no variation with $r$ of the excess leakage of heat in that region, and it is therefore clear physically that this region cannot affect the stability of the star. Thus, if $M_{a}$ denotes the mass of the part of the star where the method of perturbations is strictly applicable (i.e., the mass interior to the surface where $T \simeq 6 T_{e}$ ), then we are likely to obtain results not very far from the truth if, in our condition of stability, we extend the integration from $\circ$ to $M_{a}$ instead of from $\circ$ to $M$. In this paper we intend to apply this condition of stability to different cases.

2. To investigate the stability of the star for its fundamental mode of vibration we have to evaluate $A_{0}$. For laws of generation of energy of the form now admitted there will be a convective core at the center of the star. We can further suppose that the whole generation of energy takes place in the convective core. Now, $A_{0}$ will consist of two parts, a part $A_{C}$ due to the convection in the core and a part $A_{R}$ due to the generation of energy and the radiative flux. Following the procedure of T. G. Cowling and using the definition of $\epsilon_{2}, \epsilon_{3}, F_{2}, F_{3}$ and the values of $\bar{C}_{r}$ and $\overline{\rho E C}_{r}$ given in I, as well as the relation

$$
\frac{\delta \beta}{\beta}=\left(\Gamma_{3}-\Gamma_{\mathrm{x}}\right) \frac{\delta \rho}{\rho},
$$

which is valid for an adiabatic change, we can express $A_{C}$ in the following form:

$$
\left.\begin{array}{rl}
A_{C}=\int_{0}^{m_{c}} \frac{\delta r}{r} \cdot \frac{\delta \rho}{\rho} \cdot\left(\frac{5}{3}-\Gamma_{3}\right) & \frac{A q^{3}}{l}\left\{\mathrm{I}-\frac{\sigma r^{3}}{G m}+\mathrm{I} 2 \cdot \frac{\Gamma_{3}-\Gamma_{\mathrm{I}}}{4-3 \beta}\right\} d m \\
& +\int_{0}^{m_{C}} \frac{\delta \rho}{\rho} \cdot \frac{d \Gamma_{3}}{d m}\left\{\delta\left(4 \pi r^{2} F_{3}\right)+\delta\left(4 \pi \operatorname{Pr}^{2} \bar{C}_{r}\right)\right\} d m,
\end{array}\right\}
$$

where $m_{C}$ is the mass of the convective core.

If the radiation pressure is negligible, $\Gamma_{3}=\Gamma_{I}=\gamma=$ constant, and this expression reduces to

$$
A_{C}=-\left(\gamma-\frac{5}{3}\right) \int_{0}^{m_{C}} \frac{\delta r}{r} \cdot \frac{\delta \rho}{\rho} \cdot \frac{A q^{3}}{l}\left\{\mathrm{I}-\frac{\sigma r^{3}}{G m}\right\} d m
$$

which is the formula given by Cowling. In the core it is certainly a fair approximation to put $\gamma=\frac{5}{3}$, and we can also consider that $\delta r / r$ and $\delta \rho / \rho$ are practically constant in

${ }^{6}$ Cf. Eddington, Internal Constitution of the Stars, p. I98, I926; J. J. M. Reesink, M.N., 87, 4I4, I927; and S. Rosseland, op. cit., p. 47, n. 2.

7 Op. cit., sec. 7 , n. 2 . 
it. Then if we evaluate explicitly $d \Gamma_{3} / d m,\left(\Gamma_{3}-\Gamma_{\mathrm{r}}\right), \delta\left(4 \pi r^{2} F_{3}\right), \delta\left(4 \pi \operatorname{Pr}^{2} \bar{C}_{r}\right)$, using the expression for $\bar{C}_{r}$ and $F_{3}=\overline{\rho E C}_{r}$ given in I, and further suppose (following Cowling) that $\epsilon_{2}=A q^{3} / l$ is of the same order as $\epsilon_{\mathrm{I}}$ we find

$$
\left.\begin{array}{rl}
A_{C}= & L\left(\frac{\delta r}{r}\right)_{C}\left(\frac{\delta \rho}{\rho}\right)_{C} \\
& \times \frac{8(\mathrm{I}-\beta)}{24-2 \mathrm{I} \beta}\left[\mathrm{I}-\frac{\sigma^{2} \gamma^{3}}{G m}+\frac{\mathrm{I} 2 \beta\left(4 \cdot 5 \beta^{4}+\mathrm{IO} \cdot 5 \beta^{3}-43^{2} \beta^{2}+576 \beta-256\right)}{(8-7 \beta)^{2}(4-3 \beta)\left(32-3 \beta^{2}-24 \dot{\beta}\right)}\right]
\end{array}\right\}
$$

where the bar indicates a mean value of the quantity under it, in the convective core. * The rest of $A_{0}$ is

$$
A_{R}=\int_{0}^{m_{C}} \frac{\delta \rho}{\rho}\left(\Gamma_{3}-\mathrm{I}\right) \delta \epsilon_{\mathrm{I}} d m-\int_{0}^{M_{a}} \frac{\delta \rho}{\rho}\left(\Gamma_{3}-\mathrm{I}\right) \frac{d}{d m} \delta\left(4 \pi r^{2} F_{\mathrm{I}}\right) d m .
$$

If the rate of generation of energy is of the form

$$
\epsilon_{\mathrm{I}}=\epsilon_{\mathrm{I}}^{\mathrm{o}} \rho T^{\nu}
$$

the first term on the right-hand side of equation (4) can be written as

$$
\left(A_{R}\right)_{\epsilon}=L\left(\frac{\delta \rho}{\rho}\right)_{C}^{2}\left\{\frac{\overline{8-6 \beta}}{24-2 \mathrm{I} \beta}+\nu \overline{\left(\frac{8-6 \beta}{24-2 \mathrm{I} \beta}\right)^{2}}\right\}_{C} .
$$

The second term of $A_{R}$, due to the flux of radiation, is

$$
\left(A_{R}\right)_{F_{\mathrm{I}}}=-\left[\frac{\delta \rho}{\rho}\left(\Gamma_{3}-\mathrm{I}\right) \delta\left(4 \pi r^{2} F_{\mathrm{I}}\right)\right]_{0}^{M_{a}}+\int_{0}^{M_{a}} \delta\left(4 \pi r^{2} F_{\mathrm{I}}\right) \frac{d}{d m}\left[\left(\grave{\Gamma}_{3}-\mathrm{I}\right) \frac{\delta \rho}{\rho}\right] d m .
$$

If the law of opacity is given by

$$
\kappa=\kappa_{0} \rho^{n} T^{-(3+8)}
$$

we have

$$
\left.\begin{array}{l}
\frac{\delta\left(4 \pi r^{2} F_{\mathrm{I}}\right)}{4 \pi r^{2} F_{\mathrm{I}}}=4 \frac{\delta r}{r}\left[\mathrm{I}-\frac{\Gamma_{3}-\mathrm{I}}{\Gamma_{\mathrm{I}}}\left(\frac{\sigma^{2} r^{3}}{G m}+4\right) F\right] \\
\quad-\frac{\delta \rho}{\rho}\left[n-(3+s)\left(\Gamma_{3}-\mathrm{I}\right)\right]+4 \frac{\delta \rho}{\rho}(\mathrm{I}-F) \\
\quad \times\left\{\frac{\beta^{2}[57(\gamma-\mathrm{I})-7]-\beta[\mathrm{I} 20(\gamma-\mathrm{I})-\gamma]+64(\gamma-\mathrm{I})}{[\beta+\mathrm{I} 2(\mathrm{I}-\beta)(\gamma-\mathrm{I})]\left[\mathrm{I} 6(\gamma-\mathrm{I})-\mathrm{I} 2 \beta(\gamma-\mathrm{I})-\beta^{2}(3 \gamma-4)\right]}\right\},
\end{array}\right\}
$$

where

$$
F=\frac{\mathrm{I}}{4} \cdot \frac{d(\log P)}{d(\log T)} \quad \text { and } \quad \sigma=\frac{2 \pi}{\tau}
$$


if $\tau$ is the period of oscillation. In the region in convective equilibrium

$$
F_{C}=\frac{\mathrm{I}}{4} \cdot \frac{\Gamma_{\mathrm{I}}}{\Gamma_{3}-\mathrm{I}}
$$

In the region in radiative equilibrium, since there is no generation of energy, $4 \pi r^{2} F_{I}=L$, and we have

$$
F_{R}=\frac{4 \pi c G m(r) T^{3+s}(\mathrm{I}-\beta)}{\kappa_{0} \rho^{n} L} .
$$

Thus $\left(A_{R}\right)_{F_{\mathrm{r}}}$ can be divided into two parts, one for the region in convective equilibrium and one for the radiative envelope. It is obvious a priori (and numerical evaluations confirm it) that the first one must always remain very small so that we can neglect it. Then, if we assume that $\gamma$ is also equal to $\frac{5}{3}$ in the radiative envelope, we can write

$$
\left(A_{R}\right)_{F_{\mathrm{I}}}=-L\left(\frac{\delta \rho}{\rho} \cdot \frac{8-6 \beta}{24-2 \mathrm{I} \beta} \cdot Y\right)_{m_{c}}^{M_{a}}+L \int_{m_{c}}^{M_{a}} Y \frac{d}{d m}\left(\frac{\delta \rho}{\rho} \cdot \frac{8-6 \beta}{24-2 \mathrm{I} \beta}\right) d m,
$$

where $Y$ is the second member of equation (9) in which $\gamma=\frac{5}{3}$,

$$
\begin{aligned}
Y=4 \frac{\delta r}{r} & \left\{\mathrm{I}-\frac{8-6 \beta}{32-24 \beta-3 \beta^{2}}\left(\frac{\sigma^{2} r^{3}}{G m}+4\right) F_{R}\right\}+4 \frac{\delta \rho}{\rho}\left(\mathrm{I}-F_{R}\right) \\
& \times \frac{2\left[93 \beta^{2}-2 \mathrm{I} 6 \beta+\mathrm{I} 28\right]}{(24-2 \mathrm{I} \beta)\left(32-24 \beta-3 \beta^{2}\right)}-\frac{\delta \rho}{\rho}\left[n-(3+s)\left(\frac{8-6 \beta}{24-2 \mathrm{I} \beta}\right)\right] .
\end{aligned}
$$

In this manner we can write

$$
A_{\mathrm{o}}=\left(A_{R}\right)_{\epsilon}+A_{C}+\left(A_{R}\right)_{F_{\mathrm{I}}}
$$

the different terms being given by equations (6), (3), and (I2). The exponents of $T$ and of $\rho$ in $\epsilon$ appear explicitly in $\left(A_{R}\right)_{\epsilon}$, and those in $\kappa$ appear in $\left(A_{R}\right)_{F_{\mathrm{r}}}$. The problem is to verify whether, for likely values of these exponents, stars of different masses are stable.

3. If we suppose that $n$ and $s$ in the law of opacity are fixed and are equal, respectively, to $\mathrm{I}$ and 0.5 , for a given star we can determine the critical $\nu_{c}$ beyond which this star becomes unstable by the equation

$$
A_{0}=0 \text {. }
$$

Cowling ${ }^{8}$ solved equation ( ${ }_{5}$ ) by an approximate method for a star of small mass $\left(p_{R} \ll p_{G}\right)$, taking $\gamma=\frac{5}{3}$, and found $\nu_{c}=3900$. But he reduced this value very much (to $\sim 45^{\circ}$ ) by supposing that the sources of energy are perhaps not all concentrated in the convective core. In view of our present knowledge of the source of stellar energy this reduction in $\nu_{c}$ does not seem to be justified, and we should consider the first number as more characteristic of the problem. Performing the integration in $A_{0}$ by numerical methods and supposing that $M_{a}$ corresponds to a radius of $0.982 R$, we obtain $\nu_{c}=3600$. That this $\nu_{c}$ is smaller than the lower limit of Cowling is due to the

${ }^{8}$ Op. cit., p. 6o, n. 3 . 
fact that his process for evaluating $\nu_{c}$ corresponds to an integration extended up to the surface of the star. If we had extended our integration to $M$ (instead of only to $M_{a}$ ) we should have obtained $\nu_{c}=4050$.

In I, we have integrated the equation of pulsation for a star built on a model calculated by $\mathrm{J}$. Wasiutynski in which $p_{R}$ is not neglected. At the center of this model $\beta_{c}=0.8$ and corresponds to a mass equal to I0.263M $\odot$, if the mean molecular weight $\bar{\mu}$ is taken to be unity. As a result of this integration we found

$$
\frac{\sigma^{2} r^{3}}{G m}=0.1334 \frac{x^{3}}{\omega},
$$

if $\omega=m / m_{0}$ and $x=r / r_{0}$,

$$
m_{0}=\frac{8}{5} \cdot \pi \rho_{c} r_{0}^{3}, \quad r_{0}^{2}=\frac{5}{8} \cdot \frac{R T_{c}}{\pi G \vec{\mu} \rho_{c}}
$$

Further, the values derived for $\delta r / r, \delta \rho / \rho$ are tabulated in Table I. Here we have supposed that the adiabatic approximation is valid for all $r \leqslant 0.980 R$.

\begin{tabular}{|c|c|c|c|c|c|}
\hline$\frac{r}{r_{0}}$ & $\frac{\delta r}{r}$ & $-\frac{\delta \rho}{\rho}$ & $\frac{r}{r_{0}}$ & $\frac{\delta r}{r}$ & $-\frac{\delta \rho}{\rho}$ \\
\hline $0.0 \ldots \ldots \ldots$ & I.O & 3.0 & $4.126 \ldots \ldots$ & I. 548 & $6.6 \mathrm{I}$ \\
\hline $0.4 \ldots \ldots \ldots \ldots$ & I.O & 3.0 & $4.682 \ldots \ldots \ldots$ & I. 775 & 8.14 \\
\hline$\ldots \ldots$ & I . .004 & $3.01 \mathrm{I}$ & $5.286 \ldots \ldots$ & 2.14 & IO. I 2 \\
\hline I. $2 \ldots \ldots \ldots \ldots$ & I .OI I & $3 \cdot 128$ & $5.927 \ldots \ldots$ & 2.68 & I3.68 \\
\hline I.6........... & I. 040 & $3.28 \mathrm{I}$ & $6.583 \ldots$ & $3 \cdot 43$ & I9. $5 \mathrm{I}$ \\
\hline 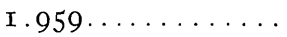 & I. .066 & $3 \cdot 398$ & $7.208 \ldots \ldots$ & $4 \cdot 49$ & $28.6 \mathrm{I}$ \\
\hline $2.274 \ldots \ldots \ldots \ldots$ & I .099 & $3 \cdot 57$ & $7.945 \ldots \ldots$ & 6.26 & 45.0 \\
\hline $2.699 \ldots$ & I. I 57 & $3 \cdot 96$ & $8.045 \ldots$ & 6.60 & 48.0 \\
\hline $3.143 \ldots \ldots \ldots$ & I. 252 & $4 \cdot 58$ & 8.2 II $\ldots \ldots \ldots$ & 7.20 & $56 \cdot 5$ \\
\hline 3.6 I $5 \ldots \ldots \ldots$ & I. 382 & $5 \cdot 39$ & & & \\
\hline
\end{tabular}

TABLE 1

In this case the mean value of the quantity under the bar in equation (3) is equal to - I.3 and $A_{C}$ is equal to $3.9 L$. The mean values occurring in equation (6) are equal to 0.46 and $0.2 \mathrm{II}$, respectively. For this model the quantity $F_{R}$ in the radiative envelope is given by

$$
F_{R}=8.7 \mathrm{I} 6 \omega \cdot \tau^{0.5} \frac{(\mathrm{I}-\beta)^{2}}{\beta}
$$

( $\tau$ corresponds here to the temperature) and is nearly equal to $\mathrm{I}$ in all this region, so that the expression for $Y$ reduces to

$$
Y=4 \frac{\delta r}{r}\left\{\mathrm{I}-\frac{8-6 \beta}{32-24 \beta-3 \beta^{2}}\left[\frac{\sigma^{2} r^{3}}{G m}+4\right]\right\}-\frac{\delta \rho}{\rho}\left[\mathrm{I}-3 \cdot 5 \cdot \frac{8-6 \beta}{24-2 \mathrm{I} \beta}\right] .
$$

A numerical integration for $\left(A_{R}\right)_{F_{x}}$ then gives

$$
\left(A_{R}\right)_{F_{\mathrm{I}}}=-\mathrm{II} 50+450=-700 \text {. }
$$


Finally, combining these results, we obtain

$$
A_{\mathrm{o}}=L\{4.14+\mathrm{I} .899 \nu+3.9-700\}
$$

The critical $\nu_{c}$ obtained in solving $A_{0}=0$ is

$$
\nu_{c}=370
$$

4. The example considered in section 3 indicates that $\nu_{c}$ decreases very rapidly when the mass increases. For a given $\nu$ it would be interesting to determine the critical mass $M_{c}$ for which the instability sets in. Unfortunately, up to the present we know the correct distribution of density for laws of generation of energy of the type considered only for the two models studied. On the other hand, if we use the standard model, its greater central condensation will lead to higher values of $\delta r / r$ near the surface; further, the fact that $\beta$ remains constant (instead of tending toward $\circ$ in the outer layers $[r=\mathrm{I}, s=0.5]$ ) will also tend to increase the stability of the star; we shall therefore be led to values of $\nu_{c}$ and $M_{c}$ which are a little too large. For this model, ${ }^{9}$ we have approximate solutions for $\delta r / r$, for different values of $a=\left(3-4 / \Gamma_{\mathrm{r}}\right)$ and $\gamma=\frac{5}{3}$; these correspond to stars of different masses. Since these models have no convective cores, $A_{0}$ is given by

$$
A_{0}=\int_{0}^{M_{a}} \frac{\delta \rho}{\rho}\left(\Gamma_{3}-\mathrm{I}\right) \delta\left\{\epsilon_{\mathrm{I}}-\frac{d}{d m}\left(4 \pi r^{2} F_{\mathrm{I}}\right)\right\} d m=\left(A_{R}\right)_{\epsilon_{\mathrm{I}}}+\left(A_{R}\right)_{F_{\mathrm{I}}}
$$

Since $\beta$ is constant and $\epsilon$ is appreciable ( $\nu$ large) only in a small region in the neighborhood of the center where $\delta \rho / \rho$ is nearly constant, we can write

$$
\left(A_{R}\right)_{\epsilon_{\mathrm{I}}}=\left(\frac{\delta \rho}{\rho}\right)_{c}^{2}\left(\Gamma_{3}-\mathrm{I}\right)\left\{\mathrm{I}+\nu\left(\Gamma_{3}-\mathrm{I}\right)\right\} L
$$

In this case $F_{R}$ is exactly equal to I, and $Y$ is still given by equation ( $3^{\prime}$ ). Furthermore, the region where $4 \pi r^{2} F_{I}$ is less than $L$ is very small and near the center. Hence, $\left(A_{R}\right)_{F_{\mathrm{r}}}$ can be written in a fair approximation as

$$
\begin{aligned}
\left(A_{R}\right)_{F_{\mathrm{I}}}=- & \left(\Gamma_{3}-\mathrm{I}\right) L \int_{0}^{M_{a}} \frac{\delta \rho}{\rho} \\
& \cdot \frac{d}{d m}\left\{\left[\mathrm{I}-\frac{\Gamma_{3}-\mathrm{I}}{\Gamma_{\mathrm{I}}}\left(\frac{\sigma^{2} r^{3}}{G m}+4\right)\right] 4 \frac{\delta r}{r}-\frac{\delta \rho}{\rho}\left[n-(3+s)\left(\Gamma_{3}-\mathrm{I}\right)\right]\right\} d m .
\end{aligned}
$$

If we write

$$
\sigma^{2}=\frac{4 \pi G}{3} \cdot \frac{\left(3 \Gamma_{\mathrm{I}}-4\right)}{2.0 \mathrm{I} 8 \mathrm{I} 3} \cdot \bar{\rho} \Sigma^{2}
$$

9 P. Ledoux and C. L. Pekeris, Ap.J., 94, I 24, I94I. 
we can express $\left(A_{R}\right)_{F_{\mathbf{x}}}$ in the following form:

$$
\begin{aligned}
\frac{\left(A_{R}\right)_{F_{\mathrm{I}}}}{\left(\Gamma_{3}-\mathrm{I}\right) L}= & -\left\{\frac{\delta \rho}{\rho}\left[4-\frac{\Gamma_{3}-\mathrm{I}}{\Gamma_{\mathrm{I}}}\left(\frac{4 a \Gamma_{\mathrm{I}} \Sigma^{2}}{2.0 \mathrm{I} 8 \mathrm{I} 3} \cdot \frac{\bar{\rho}(R)}{\bar{\rho}(r)}+\mathrm{I} 6\right)\right] \frac{\delta r}{r}\right. \\
& \left.\left.-\frac{n-(3+s)\left(\Gamma_{3}-\mathrm{I}\right)}{2}\left(\frac{\delta \rho}{\rho}\right)^{2}\right\}_{0}^{M_{a}}+\left(\mathrm{I}-4 \cdot \frac{\Gamma_{3}-\mathrm{I}}{\Gamma_{\mathrm{I}}}\right)_{4} \int_{0}^{M_{a}} \frac{\delta r}{r}\right\} \\
& \cdot \frac{d}{d m}\left(\frac{\delta \rho}{\rho}\right) d m-\frac{\Gamma_{3}-\mathrm{I}}{\Gamma_{\mathrm{I}}} \cdot \frac{\Gamma_{\mathrm{I}} 4 a \Sigma^{2}}{2.0 \mathrm{I} 8 \mathrm{I} 3} \int_{0}^{M_{a}} \frac{\bar{\rho}(R)}{\bar{\rho}(r)} \cdot \frac{\delta r}{r} \cdot \frac{d}{d m}\left(\frac{\delta \rho}{\rho}\right) d m .
\end{aligned}
$$

Although the levels where the temperature is equal to $6 T_{e}$ are not at homologous points in stars of different masses, we shall stop the integration at $r_{a}=0.985 R$ for all the stars. If we suppose that $n=\mathrm{I}, s=0.5\left(\kappa=\kappa_{0} \rho T^{-3.5}\right)$, the numerical evaluation of $A_{0}$ gives the critical $\nu_{c}$ contained in the fourth column of Table 2 .

TABLE 2

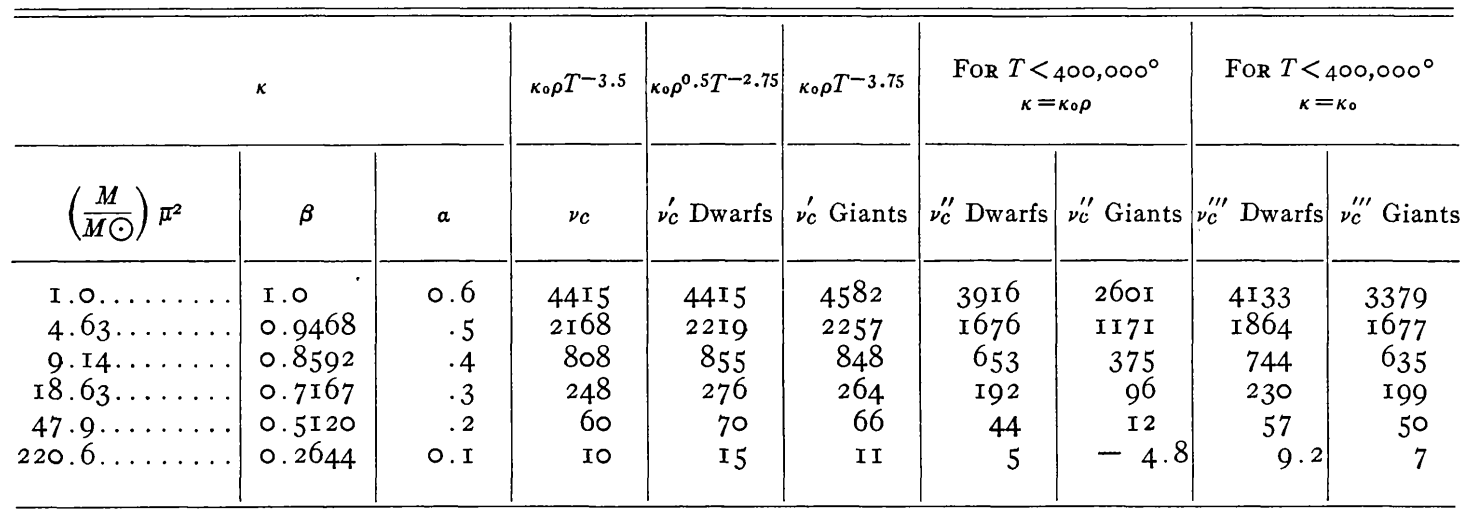

Their dependence upon the mass is illustrated by Figure I (solid curve). The dashed curve is extrapolated from the two $\nu_{c}$ 's obtained with Cowling's model. From this figure, given a $\nu$, we can always determine the critical mass $M_{c}$ beyond which instability sets in. For instance, for $\nu=\mathrm{I} 6$ and $\bar{\mu}=\mathrm{I}$, we obtain $M_{c}=\mathrm{I} 28 M_{\odot}$. Probably if we had used a correct model (with a convective core), we should have obtained something like Iо०M $M_{\odot}$.

5. From the work of B. Strömgren ${ }^{\mathrm{I0}}$ and more recently of P. M. Morse ${ }^{\mathrm{II}}$ it appears that we can roughly take the influence of the guillotine factor into account by replacing $\kappa=\kappa_{0} \rho T^{-3.5}$ by $\kappa=\kappa_{0} \rho^{0.5} T^{-2.75}$ for the stars of the main sequence and by $\kappa=\kappa_{0} \rho T^{-3.75}$ in the case of the giants. ${ }^{12}$ In general, the change $\Delta\left(A_{R}\right)_{F_{\mathrm{x}}}$ corresponding to the changes $\Delta n$ and $\Delta s$ in the exponents of $\rho$ and $T$ in $\kappa$ is given by

$$
\frac{\Delta\left(A_{R}\right)_{F_{\mathrm{I}}}}{\left(\Gamma_{3}-\mathrm{I}\right) L}=\left[\Delta n-\left(\Gamma_{3}-\mathrm{I}\right) \Delta s\right] \frac{\mathrm{I}}{2}\left[\left(\frac{\delta \rho}{\rho}\right)^{2}\right]_{0}^{M_{a}} .
$$

го Zs.f. Ap., 4, Ir8, r932; 7, 222, т933.

II $A$ p.J., 92, 27, I940.

${ }_{12}$ These laws are obtained in keeping one of the variables $\rho$ (or $T$ ) constant and trying to represent the variation of $\kappa$ in that case as a function of the other $T$ (or $\rho$ ). As Dr. Chandrasekhar has pointed out to me, this procedure is likely to lead to errors, and we should rather have to investigate the changes in $\kappa$ for the simultaneous variations of $\rho$ and $T$, which take place in the particular star studied. But here we want mainly to get some idea as to the importance of the changes in the stability which correspond to given changes in $\kappa$. 
Using this, we obtain for the cases considered, values of $\nu_{c}^{\prime}$ tabulated in the fifth and sixth columns of Table 2 . We notice that the stability remains practically unaffected.

However, the calculations by Morse are concerned only with the case of temperatures higher than $4 \times 10^{5}$ degrees, and for smaller values important changes in $\kappa$ are not impossible. To gain some idea as to the effects of these changes on the stability, we have considered the two following cases: $\kappa \propto \rho$ and $\kappa=$ constant for $T<400,000^{\circ}$. The corresponding changes in $\left(A_{R}\right)_{F_{\mathrm{r}}}$ are still given by equation (I8), where the lower limit 0 has to be replaced by the radius at which the temperature of $4 \times 10^{5}$ degrees is reached. For the stars of the main sequence this happens somewhere at $r=6.5 R / 6.9$, if we take a central temperature of the order of $2 \times$ IO $^{7}$ degrees, while for the giants $T<4 \times 10^{5}$ for $r \geqslant 5.5 R / 6.9$. The $\nu_{c}^{\prime \prime}$ and $\nu_{c}^{\prime \prime \prime}$ obtained in this way are given in the last columns of

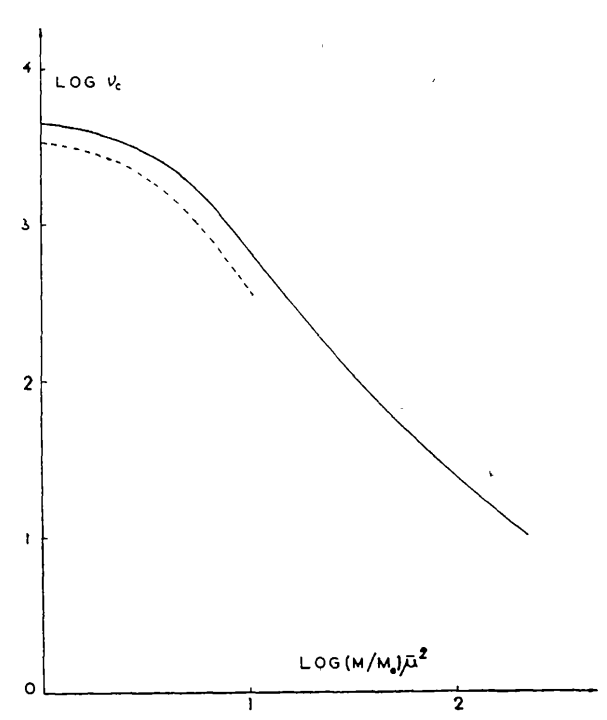

FIG. I

Table 2. Their values show that the modifications in $\kappa$ considered, especially the case $\kappa=\kappa_{0} \rho$, reduce appreciably the stability of the starparticularly in the case of the giants. This is due to the fact that the region where the changes take place extends more deeply in the giants than in the dwarfs. However, for likely laws of generation of energy, the critical mass, even for the giants, still remains high (for $\nu=\mathrm{I} 8,\left[M / M_{\odot}\right] \bar{\mu}^{2}$ $\sim 40$ ).

6. Thus far we have neglected the energy of ionization and excitation. If we take this into account, the adiabatic exponents $\Gamma_{\mathrm{I}}$ and $\Gamma_{3}$ are simply replaced by new ones, $\Gamma_{x}^{\prime}$ and $\Gamma_{3}^{\prime}$. From computations of $\Gamma_{\mathrm{I}}^{\prime}$ made by Fowler and Guggenheim $^{\mathrm{I} 3}$ for different elements and temperatures greater than $10^{6}$ degrees and from the fact that we now adopt a very high abundance of hydrogen we can conclude that in the main part of the stars $\left(T>10^{6}\right.$ degrees $) \Gamma^{\prime}$ is practically the same as $\Gamma$.

For discussing the stability of the radiative equilibrium in the region with temperatures below IO $^{6}$ degrees, Biermann ${ }^{14}$ has developed an approximate method of evaluating $\Gamma_{x}^{\prime}$ for the lower temperatures in the case of a mixture of elements. One of his mixtures is given in Table 3 .

For this mixture and for a star of small mass $\left(p_{R} \ll P\right)$, Biermann found that in the range of temperatures from $6 \times{ }^{10} 0^{4}$ degrees to $4 \times 10^{5}$ degrees the mean value of $\Gamma_{\mathrm{I}}^{\prime}$ is I.43. For temperatures between $4 \times 10^{5}$ degrees and $10^{6}$ degrees, the differences in $\left(\Gamma_{\mathrm{I}}^{\prime}-\Gamma_{\mathrm{I}}\right)$ are already much smaller, and we shall neglect them here. As the mass of the star increases, the upper limit of the interesting range of temperatures decreases, for, $p_{R}$ becoming more important, a given temperature corresponds to a smaller density and this favors higher ionization. We have determined these limits and the mean values of $\Gamma_{I}^{\prime}$ for the same mixture as Biermann, for different masses. Our results are contained in columns 2 and 3 of Table 4.

For the case of one element $z$ in the $r$ and $(r+I)$ states of ionization, with a difference of energy $\chi_{r}^{z}$ between them, the expression for $\Gamma_{3}^{\prime}$ is

$$
\Gamma_{3}^{\prime}=\mathrm{I}+\frac{\left[\left(\frac{\partial N}{\partial T}\right)_{\rho} \cdot \frac{T}{\left(N+M_{z}\right)}+\frac{4}{\beta}-3\right]}{\frac{8}{2}+\mathrm{I} 2 \frac{(\mathrm{I}-\beta)}{\beta}+\left[\frac{3}{2}+\frac{\chi_{r}^{z}}{k T}\right] \frac{T}{N+M_{z}} \cdot\left(\frac{\partial N}{\partial T}\right)_{\rho}},
$$

×3 M.N., 85, 961, 1925.

${ }^{{ }^{4}}$ A.N., 259, $221,1936$. 
where $M_{z}$ and $N$ are the numbers of atoms and free electrons. Applying the method of Biermann, it is easy to derive a practical form for $\Gamma_{3}^{\prime}$ in the case of a mixture of elements. The interesting range of temperatures is about the same for $\Gamma_{3}^{\prime}$ as for $\Gamma_{1}^{\prime}$, and the fourth column of Table 4 contains the values of $\left(\Gamma_{3}^{\prime}-\mathrm{I}\right)$. Although the radius corresponding to the upper limit of the range of temperatures varies a little with the mass, especially in the dwarfs (for the giants the diminution of the central temperature, when $M$ increases, compensates this effect), we have adopted as before constant values for this radius equal to $6.5 R / 6.9$ for the dwarfs and $5.5 R / 6.9$ for the giants. Introducing the values of $\Gamma_{\mathrm{I}}^{\prime}$ and $\Gamma_{3}^{\prime}$ in the part of the condition of stability corresponding to the region considered, we find the values of $\nu_{c}$ contained in the fifth and sixth columns of Table 4 .

We see that changes in the $\Gamma$ as in $\kappa$, when confined to a region of the star whose limits depend upon physical conditions, affect the stability of the giants more than that of the dwarfs. If we combine the first change in $\kappa(\Delta n=0, \Delta s=-3.5)$ and the preced-

TABLE 3

\begin{tabular}{c|c|c|c|c}
\hline Element & $H$ & $O$ & $M g$ & $F e$ \\
\hline No. of atoms.... & I 27 & 8 & 3 & $\mathrm{I}$ \\
\hline Weight........ & $\mathrm{I} 28$ & $\mathrm{I} 28$ & 73 & 56 \\
\hline
\end{tabular}

TABLE 4

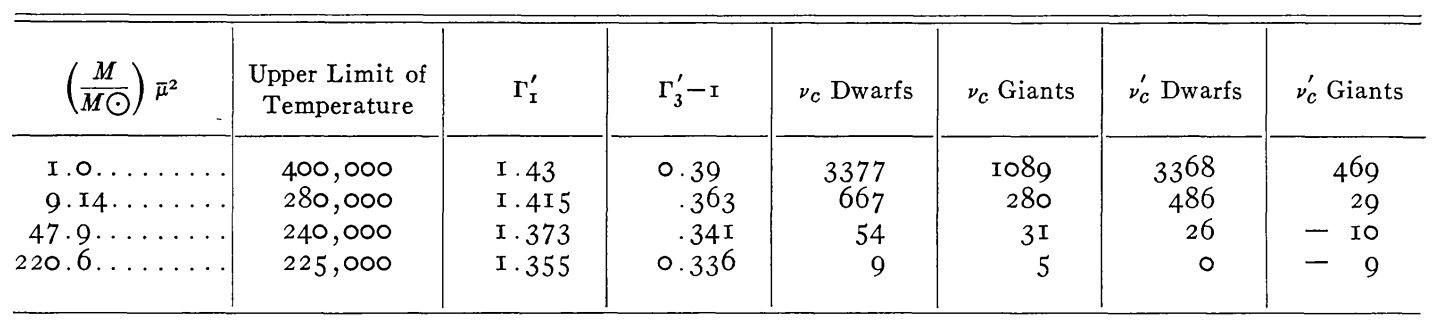

ing changes in $\Gamma$ we obtain the $\nu_{c}^{\prime}$ in the two last columns of Table 4. In this case a giant of a mass equal to เo $M_{\odot}$ would already be on the verge of instability for a $\nu$ equal to 18 . The small values of $\Gamma^{\prime}$ adopted in the outer part of the star will also lower the increase of the amplitude with $r$ in that region. This secondary effect will still increase the instability of the star. If the values of the $\Gamma^{\prime}$ become small enough, turbulence will appear in that region also, but it is not likely that this would increase the stability of the star, in any case not very much. ${ }^{15}$ However, the values of $\Gamma_{\mathrm{r}}^{\prime}$, etc., depend very much upon the abundances adopted for the elements. If we took an abundance in $H$ appreciably greater or allowed for the presence of $\mathrm{He}$ in appreciable quantity (already nearly completely ionized for $T={ }_{10} 0^{5}$ degrees), the region where $\Gamma_{\mathrm{r}}^{\prime}$ is different from $\Gamma_{\mathrm{r}}$ would be reduced considerably, and in general the stability of the star would increase.

7. Apart from their interest with regard to the stability of ordinary stars, these considerations are still important in their bearing upon the Cepheid phenomenon. In so far as the interior of the star is concerned, the main object of the theory of small pulsations is to provide theoretical periods in agreement with the observed ones and to explain how the star can become unstable in some of its modes of vibration so that the amplitudes of those modes can increase until they reach some limit corresponding to the

${ }^{15}$ Cf. T. G. Cowling, M.N., 98, 528, 1938. 
finite pulsations observed in the Cepheids. The comparison of the observed periods with theoretical ones for likely models indicates that it must be the fundamental mode which is responsible for the pulsation of most of the Cepheids. However, M. Schwarzschild ${ }^{16}$ has recently suggested that the first overtone is probably responsible for the pulsation in the Cepheids of type c. For the two first modes the general qualitative run of the amplitudes inside the star would be as schematized in Figure 2.

If the generation of energy is concentrated in the neighborhood of the center of the star and if the opacity decreases with compression in the whole star (this is the case for $n=\mathrm{I}, s=0.5$ and for all likely values of $\Gamma_{3}$ ), it is obvious from Figure 2 that the star is much more stable for the first mode of pulsation than for the fundamental one. But, as we have seen, in that case even the fundamental mode would be stable (except for very large masses). If we consider the changes in $\kappa$ and in $\Gamma$ in the outer regions of

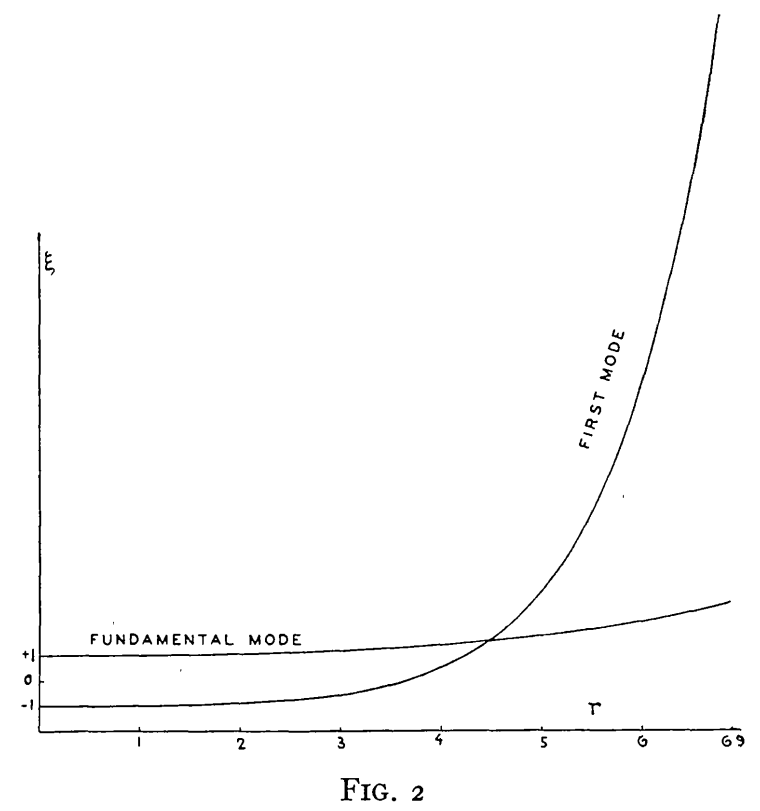

the star by which we have tried to reduce the stability of the star for the fundamental mode, it appears immediately from the run of the amplitudes that, by choosing appropriately the layer where these changes take place, they could affect the stability of the first mode of pulsation more than that of the fundamental mode. In particular, if the boundaries of this layer are determined by critical temperatures, then, as we go to Cepheids of smaller masses (shorter periods), it is very probable that this layer will recede toward the surface, the effect on the first mode becoming more important. This is in agreement with the suggestion of $M$. Schwarzschild since it concerns the Cepheids of type c. After the computations of the amplitudes for different modes announced by M. Schwarzschild have been published, an exact discussion will be possible. However, a reliable numerical integration by Miss Kluyver ${ }^{17}$ is available for the second mode of pulsation of the standard model in the case $a=0.4$, and it can be used to illustrate the situation. If we suppose that the region where the adiabatic approximation is valid has the same extension for this mode as for the fundamental one (in reality, it must be a little smaller, but not very much) and that $n=\mathrm{I}, s=0.5$ in the law of opacity, we find a value of $\nu_{c}$ of the order of $10^{6}$ if we do not take into account the possible changes of the $\Gamma$ in the outer re-

${ }^{16}$ Harvard Circ., No. 437, I940, and Pub. A.A.S., ro, No. 3, I941.

${ }^{17}$ B.A.N., No. 276 , p. $3^{1} 3$, 1936. 
gion $\left(6 \times \operatorname{IO}^{4}<T<4 \times 10^{5}\right)$ and a $\nu_{c}$ of the order $2.6 \cdot 10^{5}$ if one considers such changes, adopting the same mixture of elements as previously. Thus, in these cases the star is much more stable for the second mode than for the fundamental one. But, if one determines the $\Delta s$ in the same outer regions ( $\Delta n$ being equal to $\circ$ and $\nu$ being equal to $\mathrm{r} 8$ ) necessary to bring the star on the verge of instability for each mode, in the same two cases $\left(\Gamma\right.$ and $\left.\Gamma^{\prime}\right)$ one finds for the fundamental mode $\left(\Delta s_{f}\right)_{\Gamma}=-6.38,\left(\Delta s_{f}\right)_{\Gamma^{\prime}}=-3.66$ and for the second $\left(\Delta s_{2}\right)_{\Gamma}=-5.73$ and $\left(\Delta s_{2}\right)_{\Gamma^{\prime}}=-2.77$. In both cases this shows that for the regions chosen an appropriate change in $\kappa(\Delta s$ intermediate between the two corresponding limits $\Delta s_{f}$ and $\Delta s_{2}$ ) can make the star unstable for the second mode of vibration, although it remains stable for the fundamental mode. For a still higher mode the point where the amplitude becomes greater than that for the preceding mode will approach the surface, and it will probably occur mostly in the outer region which has very little effect on the stability of the star; and, whatever may be the changes in $\kappa$, the star will remain much more stable for this mode than for the lower mode. ${ }^{18}$

The problem is also interesting from the point of view of the generation of energy. First of all, it is well known that the ordinary nuclear reactions, which are so successful in the case of the dwarfs, fail to explain the energy production in the giants. Since the outer regions of the star cannot be the seat of generation of energy, considerations of the kind we are now concerned with cannot contribute to the solution of the question of the relative instability of different modes. Here we shall consider only the fundamental mode of pulsation. Since we cannot expect much higher $\nu$ than, say, 20, the only thing to do to decrease the stability of a star on account of the generation of energy is to suppose that it is displaced from the center toward regions where the amplitude of the pulsation is larger. "Shell-source" models satisfying this condition were considered by Gamow (resonance effect), by Gamow and Teller (light elements which would be rapidly consumed near the center), and by Öpik (hydrogen of the primitive core consumed in the course of evolution). This last hypothesis does not seem to be able to remove the difficulties as to the amount of energy generated by the giants. ${ }^{19}$

For our problem the important point is that the extension of the isothermal core will reach a maximum which it cannot outgrow. And, even when its extension is a maximum, the surface of the core is still too deep in the star for the generation of energy starting at the interface to affect very greatly the stability of the star, since the amplitudes are still too small in these central regions. In the case of Gamow another possibility is the "ballsource" model, in which $\epsilon$ is a constant in the central part of the star whose surface has a temperature $T$ equal to the temperature of resonance $T_{R}$. For such a model the distribution of density will probably not be very different from that of the standard model. In such a case the mass capable of generating energy will increase during a contraction and decrease during an expansion. For small oscillations the term $\left(A_{R}\right)_{\epsilon}$ will be

$$
\left(A_{R}\right)_{\epsilon}=-L \frac{3 \rho_{R} T_{R}}{\bar{\rho}_{R} r_{R}} \cdot\left(\frac{d r}{d T}\right)_{R} \cdot\left(\frac{\delta T}{T}\right)_{R}^{2}=-L \frac{3 \rho_{R} T_{R}}{\bar{\rho}_{R} r_{R}} \cdot\left(\frac{d r}{d T}\right)_{R} \cdot\left(\Gamma_{3}-\mathrm{I}\right)^{2}\left(\frac{\delta \rho}{\rho}\right)_{R}^{2}
$$

Assuming the distribution of density of the standard model and $a=0.4$, we have calculated the quantity $\left(A_{R}\right)_{\epsilon} /\left(\Gamma_{3}-\right.$ I $) L$ by means of equation (I9) at different depths (i.e., different $\left.T_{R}\right)$ and have compared it with $(\delta \rho / \rho)_{c}^{2}\left[\mathrm{I}+\nu_{c}\left(\Gamma_{3}-\mathrm{I}\right)\right]$ for the corresponding case (cf. Table 2, col. $4,1.4$ ). Whatever we choose for $T_{R}$, the first quantity

\footnotetext{
${ }^{18}$ If by the consideration of a special process like the one introduced recently by Eddington this outer region can actually be of great importance, it will then be difficult to escape the conclusion that higher modes rather than the fundamental or the first ones must appear. The present investigation was completed before Eddington's paper (M.N., ror, I82, I94I) reached the author.
}

${ }^{19}$ Cf., e.g., J. Wasiutynski, M.N., гоo, 362 , I940. 